OPEN ACCESS

Edited by:

Fangyuan Zhang,

Southwest University, China

Reviewed by:

Zhichao Xu,

Northeast Forestry University, China

Li Gu,

Fujian Agriculture and Forestry

University, China

*Correspondence:

Aizhong Liu

liuaizhong@mail.kib.ac.cn

Specialty section:

This article was submitted to

Plant Metabolism

and Chemodiversity,

a section of the journal

Frontiers in Plant Science

Received: 03 November 2021

Accepted: 07 December 2021

Published: 11 January 2022

Citation:

Wang Y, Tong Y, Adejobi OI,

Wang $Y$ and Liu A (2022) Research

Advances in Multi-Omics on

the Traditional Chinese Herb

Dendrobium officinale.

Front. Plant Sci. 12:808228.

doi: 10.3389/fp/s.2021.808228

\section{Research Advances in Multi-Omics on the Traditional Chinese Herb Dendrobium officinale}

\author{
Yue Wang ${ }^{1,2}$, Yan Tong ${ }^{1,2}$, Oluwaniyi Isaiah Adejobi, ${ }^{1,2}$, Yuhua Wang ${ }^{1,2}$ and Aizhong Liu ${ }^{3 *}$ \\ ${ }^{1}$ Key Laboratory of Economic Plants and Biotechnology, Yunnan Key Laboratory for Wild Plant Resources, Kunming Institute \\ of Botany, Chinese Academy of Sciences, Kunming, China, ${ }^{2}$ Bio-Innovation Center of DR PLANT, Kunming Institute \\ of Botany, Chinese Academy of Sciences, Kunming, China, ${ }^{3}$ Key Laboratory for Forest Resources Conservation \\ and Utilization in the Southwest Mountains of China, Ministry of Education, Southwest Forestry University, Kunming, China
}

Dendrobium officinale Kimura et Migo is an important epiphytic plant, belonging to the Orchidaceae family. There are various bioactive components in $D$. officinale plants, mainly including polysaccharides, alkaloids, and phenolic compounds. These compounds have been demonstrated to possess multiple functions, such as antioxidation, immune regulation, and anti-cancer. Due to serious shortages of wild resources, deterioration of cultivated germplasm and the unstable quality of $D$. officinale, the study has been focused on the biosynthetic pathway and regulation mechanisms of bioactive compounds. In recent years, with rapid developments in detection technologies and analysis tools, omics research including genomics, transcriptomics, proteomics and metabolomics have all been widely applied in various medicinal plants, including $D$. officinale. Many important advances have been achieved in $D$. officinale research, such as chromosome-level reference genome assembly and the identification of key genes involved in the biosynthesis of active components. In this review, we summarize the latest research advances in $D$. officinale based on multiple omics studies. At the same time, we discuss limitations of the current research. Finally, we put forward prospective topics in need of further study on $D$. officinale.

Keywords: Dendrobium officinale, multi-omics, bioactive compounds, biosynthesis, regulation

\section{INTRODUCTION}

Dendrobium officinale Kimura et Migo, an important epiphytic plant in the Orchidaceae family, has been used as a traditional Chinese medicine herb for thousands of years in China, and widely as a medicinal herb in many Asian countries (Ng et al., 2012). Wild D. officinale is often distributed in tropical regions, and it has been widely introduced to subtropical regions for cultivation. Generally, D. officinale can nourish the stomach, moisten the lung, relieve coughs and strengthen the body (Ng et al., 2012; Tang et al., 2017; Chen W. et al., 2021). Chemical isolation of compounds has identified diverse active compounds including polysaccharides, flavonoids, alkaloids, and multiple amino acids. In particular, the polysaccharides, alkaloids, and flavonoids extracted from $D$. officinale are thought to be the main bioactive ingredients for medicine (Meng et al., 2016; Tang et al., 2017; Wang et al., 2020; Wang, 2021). Pharmacological studies have confirmed the positive effects of polysaccharides, alkaloids and flavonoids extracted from $D$. officinale on immune regulation and 
liver protection (Wang et al., 2020), on anti-cancer and neuroprotection (Zhang et al., 2003; Wang et al., 2020), and on anti-oxidant activity (Yuan et al., 2020a). Phenols extracted from $D$. officinale (such as erianin and gigantol) are active in enhancing immune activity while also exhibiting anti-tumor and anti-oxidant properties (Zhang et al., 2018; He et al., 2020). Owning to the increasing demands for diverse healthy products based on $D$. officinale, the cultivation of $D$. officinale is expanded throughout south China and south Asian countries with an annual output value of 10 billion Yuan per year (Wang and Shi, 2019).

Studies have revealed that the content of active compounds often varies among different tissues in D. officinale. For instance, stems accumulate the most abundant polysaccharides (Wang et al., 2020; Yuan et al., 2020a), while leaves contain higher contents of flavonoids and alkaloids than other tissues (Shen et al., 2017; Chen et al., 2019; Yuan et al., 2020b; Wang, 2021). Bibenzyls (belonging to phenolic compounds) including erianin and gigantol are mainly detected in roots and stems (Adejobi et al., 2021). Moreover, increasing evidence shows that the biosynthesis and accumulation of diverse active compounds are often influenced by various environmental factors such as different cultivation substrates (Zuo et al., 2020) and habitats (Lei et al., 2018). Therefore, it is important to dissect the physiological and molecular mechanisms underlying accumulation of those active compounds during the growth and development of different tissues for breeding by genetic improvement, cultivation and management practices in agriculture. In the last decade, technical development of DNA sequencing and molecular identification has led to the extensive application of diverse omics such as genomics, transcriptomics, proteomics and metabolomics, which are used to dissect the physiological and molecular mechanisms of active compound biosynthesis. These multi-omics studies have provided vital insights into the understanding of the biosynthesis pathways of active compounds and their molecular regulation networks during the growth and development of $D$. officinale. In this review, we focus on the genetic and molecular bases of active compounds identified from $D$. officinale based on the latest research on multi-omics in D. officinale to aid more studies in the discovery of the physiological and molecular mechanisms that underlie those active compounds accumulated in D. officinale, facilitating directional breeding, cultivation and management, and medicinal usage.

\section{GENOMIC RESEARCH IN DENDROBIUM OFFICINALE}

Genome data can provide basic and comprehensive information on genetic backgrounds and bridge gaps between genetic bases and active compounds in medicinal plants. To date, three versions of $D$. officinale genomic data $(2 n=38)$ have been reported, with the genome size varying from 1.01 to $1.36 \mathrm{~Gb}$ and heterozygosity of $0.48-1.27 \%$ (Yan et al., 2015; Zhang G. Q. et al., 2016; Niu et al., 2021). A comparison between them is listed in Table 1. The first two versions were assembled at the scaffold level, while the third version was assembled onto
TABLE 1 | A quality comparison of three $D$. officinale genome versions.

\begin{tabular}{|c|c|c|c|}
\hline Items & $\begin{array}{l}\text { v.1.0 (Yan } \\
\text { et al., 2015) }\end{array}$ & $\begin{array}{l}\text { v.2.0 (Zhang } \\
\text { G. Q. et al., } \\
\text { 2016) }\end{array}$ & $\begin{array}{l}\text { v.3.0 (Niu } \\
\text { et al., 2021) }\end{array}$ \\
\hline $\begin{array}{l}\text { Sequencing } \\
\text { platform }\end{array}$ & $\begin{array}{c}\text { Illumina HiSeq } \\
2000 \text { PacBio } \\
\text { RS II }\end{array}$ & $\begin{array}{c}\text { Illumina HiSeq } \\
2000\end{array}$ & $\begin{array}{c}\text { Illumina HiSeq } \\
2500 \text { PacBio } \\
\text { Sequel II }\end{array}$ \\
\hline Genome size & $1.36 \mathrm{~Gb}$ & $1.01 \mathrm{~Gb}$ & $1.23 \mathrm{~Gb}$ \\
\hline Heterozygosity & $0.48 \%$ & $0.628 \%$ & $1.27 \%$ \\
\hline Contig N50 & $25.12 \mathrm{~kb}$ & $33.09 \mathrm{~kb}$ & $1.44 \mathrm{Mb}$ \\
\hline Contig number & 814,881 & 105,732 & 2,430 \\
\hline Scaffold N50 & $76.49 \mathrm{~kb}$ & $391.46 \mathrm{~kb}$ & $63.07 \mathrm{Mb}$ \\
\hline Assembly level & Scaffold & Scaffold & Chromosome \\
\hline WGD & - & 2 & 2 \\
\hline Repetitive ratio & $63.33 \%$ & $78.1 \%$ & $64.39 \%$ \\
\hline SNPS & $5,432,657$ & $5,758,781$ & - \\
\hline $\begin{array}{l}\text { Protein-coding } \\
\text { genes }\end{array}$ & 35,567 & 28,910 & 27,631 \\
\hline $\begin{array}{l}\text { Specific gene } \\
\text { families }\end{array}$ & 1,462 & 629 & 1,196 \\
\hline $\begin{array}{l}\text { Functionally } \\
\text { annotated genes }\end{array}$ & 34,699 & - & 25,894 \\
\hline
\end{tabular}

19 chromosomes with third-generation and $\mathrm{Hi}-\mathrm{C}$ sequencing platforms (Niu et al., 2021). The number of protein-coding genes varied from 27631 to 35567, with 629-1462 gene families specific to $D$. officinale. There are differences in genomic data between three versions, which could result from the sequencing platform, assembly strategies and genome annotation methods. The genome of $D$. officinale characteristically features abundant repetitive sequences and two rounds of whole-genome duplication (WGD; Zhang G. Q. et al., 2016; Niu et al., 2021). In the future, with the aid of genetic and physical maps and more transcriptomic data, genome quality and annotation integrity can be further improved.

For medicinal plants, one of the most important studies in genomics is to annotate and identify candidate genes related to the biosynthesis of active compounds. Three versions of the D. officinale genome showed marked expansion of some genes encoding the enzymes responsible for the biosynthesis of active compounds. According to the earlier two versions, some key enzyme genes involved in the biosynthesis of active compounds were identified (Yan et al., 2015; Zhang G. Q. et al., 2016). Based on the third version, the researchers comprehensively identified the genes in the biosynthetic pathways of polysaccharides, alkaloids, and flavonoids, including 418 genes (Niu et al., 2021; Figures 1-3). For the biosynthesis of polysaccharides, a total of 268 genes encoding 56 enzymes were identified (Niu et al., 2021). Among them, there are 13, 10, and 15 genes that encoded cellulose synthase-like A (CLSA), sucrosephosphate synthase and sucrose synthase, respectively, which showed expansion (Yan et al., 2015; Zhang G. Q. et al., 2016). In particular, the galacturonosyltransferase and $\beta$-galactosidase gene families are uniquely evolved in D. officinale (Yan et al., 2015). The large-scale expansion of these genes may partly explain the abundant polysaccharides in D. officinale. For the 


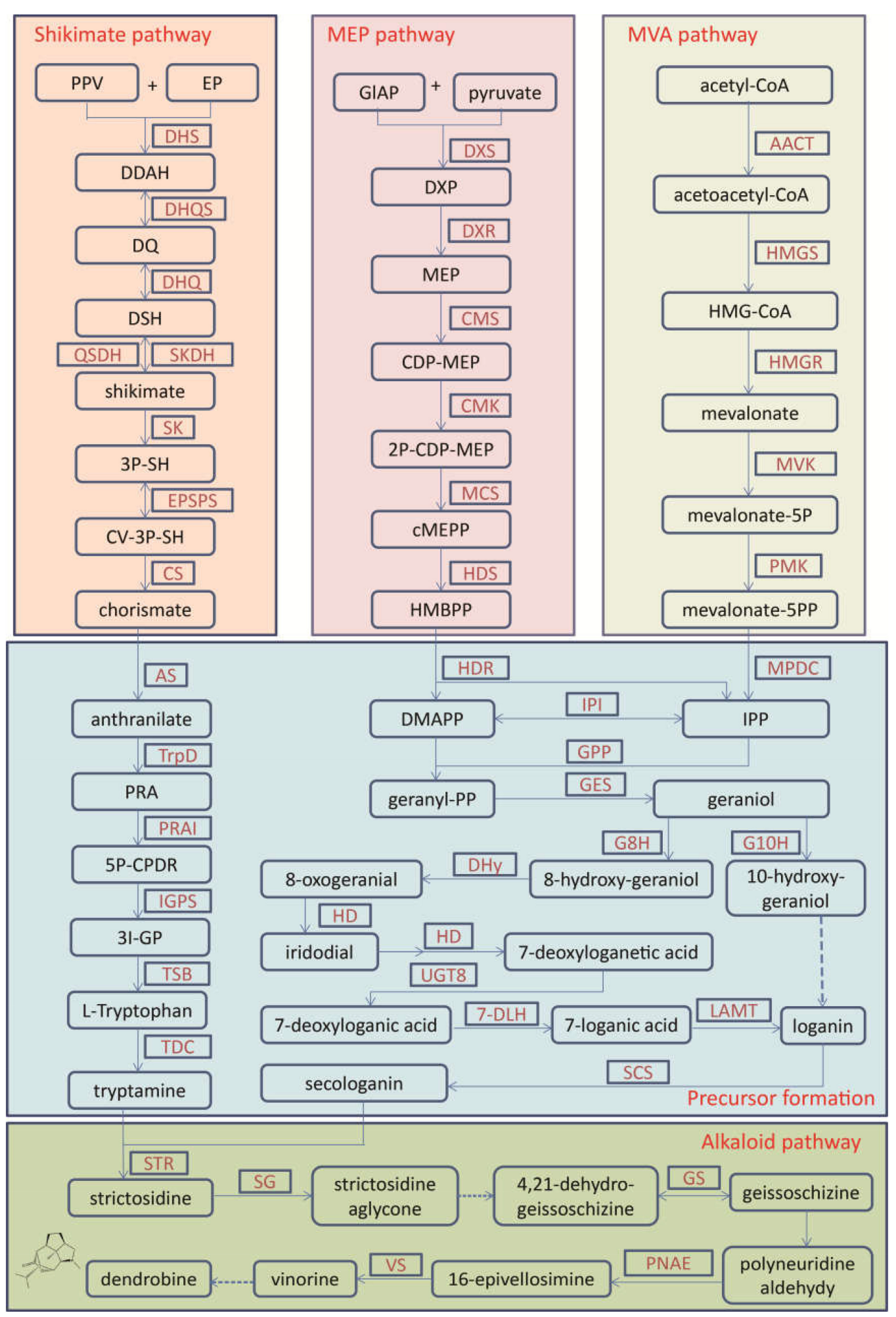

FIGURE 1 | The biosynthetic pathway for terpenoid indole alkaloids in Dendrobium officinale. The alkaloid biosynthesis contains the precursor formation from the shikimate, MEP, and MVA pathways and the alkaloid pathway. 2P-CDP-MEP, 2-phospho-4-(cytidine 5'-diphospho)-2-C-methyl-D-erythritol; 3I-GP,

(3-indoyl)-glycerolphosphate; 3P-SH, shikimate 3-phosphate; 5P-CPDRC, 1-(2-carboxyphenylamino)-1'-deoxy-D-ribulose 5-phosphate; CDP-MEP, 4-(cytidine 5'-diphoshpo)-2-C-methyl-D-erythritol; CMEPP, 2-C-methyl-D-erythritol-2,4-cyclodiphosphate; CV-5P-SH, 5-O-(1-carboxyvinyl)-3-phosphoshikimate; DDAH, 7P-2-dehydro-3-deoxy-D-arabino-heptonate; DMAPP, dimethylallyl-PP; DQ, 3-dehydroquinate; DSH, 3-dehydroshikimate; DXP, 1-deoxy-D-xylulose-5-phosphate; 
FIGURE 1 I EP, D-erythrose 4-phosphate; HMBPP, 1-hydroxy-2-methyl-2-butenyl-4-diphosphate; HMG-CoA, 3-hydroxy-3-methylglutaryl-CoA; IPP, isopentenyl-PP;
MEP, 2-C-methyl-D-erythritol-4-phosphate; PPV, phosphoenolpyruvate; PRA, N-(5-phospho- $\beta$-D-ribosyl)-anthranilate; 7-DLH, 7-deoxyloganate 7-hydroxylase;
AACT, acetyl-CoA acetyltransferase; AS, anthranilate synthase; CMK, 4-diphosphocytidyl-2C-methyl-D-erythritol kinase; CMS,
4-diphosphocytidyl-2C-methyl-D-erythritol synthase; CPR, NADPH-hemoprotein reductase; CS, chorismate synthase; DHQ, 3-dehydroquinate dehydratase; DHQS,
3-dehydroquinate synthase; DHS, 3-deoxy-7-phosphoheptulonate synthase; DHy, dehydrogenase; DXR, 1-deoxy-D-xylulose-5-phosphate reductoisomerase; DXS,
1-deoxyxylulose-5-phosphate synthetase; EPSPS, 3-phosphoshikimate 1-carboxyvinyltransferase; G1OH, geraniol 10-hydroxylase; G8H, geraniol 8-hydroxylase;
GPP, geranyl diphosphate diphosphatase; GES, geranyl diphosphate synthase; GS, geissoschizine synthase; HD, hydroxylase; HDR,
4-hydroxy-3-methylbut-2-en-1-yl diphosphate reductase; HDS, 1-hydroxy-2-methyl-2-(E)-butenyl-4- diphosphate synthase; HMGR, HMG-CoA reductase; HMGS,
HMG-CoA synthase; IGPS, indole-3-glycerolphosphate synthase; IPI, IPP isomerase; LAMT, loganate O-methyltransferase; MCS, 2-C-methyl-D-erythritol 2,4-
cyclodiphosphate synthase; MPDC, mevalonate diphosphate decarboxylase; MVK, mevalonate kinase; PMK, phosphomevalonate kinase; PNAE,
polyneuridine-aldehyde esterase; PRAI, phosphoribosylanthranilate isomerase; QSDH, quinate/shikimate dehydrogenase; SCS, secologanin synthase; SG,
strictosidine $\beta$-D-glucosidase; SK, shikimate kinase; SKDH, shikimate dehydrogenase; STR, strictosidine synthase; TDC, tryptophan decarboxylase; TrpD,
anthranilate phosphoribosyltransferase; TSB, tryptophan synthase; UGT8, 7-deoxyloganetic acid glucosyltransferase; VS, vinorine synthase.

two other types of active compounds (alkaloids and flavonoids), there are 98 and 52 genes that could be involved in the biosynthesis pathways, respectively (Niu et al., 2021). A putative enzyme (polyneuridine-aldehyde esterase) may be responsible for the extension of 16-epivellosimine (Yan et al., 2015; Figure 1). Moreover, other genes related to distinctive traits were also annotated and identified, including fungus symbiosis, drought resistance, photosynthesis, and flower development (Yan et al., 2015; Zhang G. Q. et al., 2016; Niu et al., 2021). The analysis of those genes suggests their adaptation to certain environments.

Moreover, D. officinale has distinct flower architecture, which has attracted researchers' attention. Genomic research provides important insights on flower development in D. officinale. The ABCDE model is a classical model for flower architecture. Although there are fewer MADS-box genes (63) in D. officinale than that in rice and Arabidopsis, five classes of MADS-box genes all exist, suggesting presence of complete flower genes in D. officinale (Yan et al., 2015; Zhang G. Q. et al., 2016). Unique genes, gene expansion and gene loss were observed in MADS-box gene family. Among them, MADS-box ZMM17 gene family, belong to B class, is unique in D. officinale, which could be related to the distinct flower architecture (Yan et al., 2015). ANR1, StMADS11, and MIKC* genes generally participate in regulation of growth and development, and their expansion could result in the diversity (Zhang G. Q. et al., 2016). The less number of type I MADS box genes may lead to the failure of endosperm development (Zhang G. Q. et al., 2016). The genomic investigation provides important hues for understanding the distinct and various flowers in $D$. officinale.

The genomic research of $D$. officinale provides abundant information for understanding the potential genetic resources of active component accumulation and insights for some important traits. In the future, confirming the key genes related to the above traits will be a research focus, and Genome-Wide Association Studies (GWAS) could be one of the principle methods for mapping candidate genes.

\section{TRANSCRIPTOMIC RESEARCH IN DENDROBIUM OFFICINALE}

Transcriptomic sequencing (RNA-seq) has been widely applied to research in medicinal plants including $D$. officinale due to low expenditure, high throughout, high sensitivity and no limitation from genome sequencing requirement. Since the first transcriptome of D. officinale stems was reported (Guo et al., 2013), there are now up to 30 transcriptome data of $D$. officinale available in the NCBI (Table 2). This research characterizes the biosynthetic pathways of active compounds in D. officinale, which provides an important basis for its uses as a medicinal material (Table 1; Yuan et al., 2020a). Transcriptomic analysis was also used to investigate the effects of various factors on the accumulation of active compounds, including the material location, hormones and more (Chen et al., 2019; Ren et al., 2020; Zuo et al., 2020). Moreover, the potential molecular mechanisms of a variety of physiological processes were analyzed, such as response to stress, flowering and symbiotic relationships (Wang et al., 2018; Zou et al., 2018; Li H. et al., 2021).

Tissue-specifically expressed genes can be identified through analysis of comparative transcriptomics. A total of 2645, 256, 42, and 54 transcripts were highly expressed in four organs (flower, root, leaf, and stem), respectively (Meng et al., 2016). Those differential expression genes (DEGs) were shown to be involved in organ-specific functions. Among them, eight genes with high transcripts in stem were annotated to the secondary metabolic pathways and carbohydrate transport and metabolism, which was consistent with its abundant metabolites. Moreover, 25 genes were identified to participate in the regulation of flowering, which suggests the presences of classical flowering pathways (Shen et al., 2017).

Alkaloids are one type of the earliest-identified active compounds in D. officinale (Tang et al., 2017). Although there are different types of alkaloids accumulated in D. officinale (Jiao et al., 2018), the main classes of alkaloids are terpenoid indole alkaloids (TIAs; Guo et al., 2013; Niu et al., 2021). Alkaloids are present in multiple tissues, including protocormlike bodies (PLB), flower, root, leaf and stem, and the content in PLBs was the highest (Wang et al., 2021). There were 25 genes that participated in the shikimate, mevalonate (MVA) and methylerythritol phosphate (MEP) and strictosidine pathways, and other genes were potentially related to the alkaloid synthesis, such as cytochrome P450s, aminotransferases, methyltransferases, multidrug resistance protein (MDR), transporters and transcription factors (Guo et al., 2013; Figure 1). Further expression analysis confirmed that the genes responsible for 1-deoxy-D-xylulose-5-phosphate synthase (DXS), 5-enolpyruvylshikimate-3-phosphate synthase, 


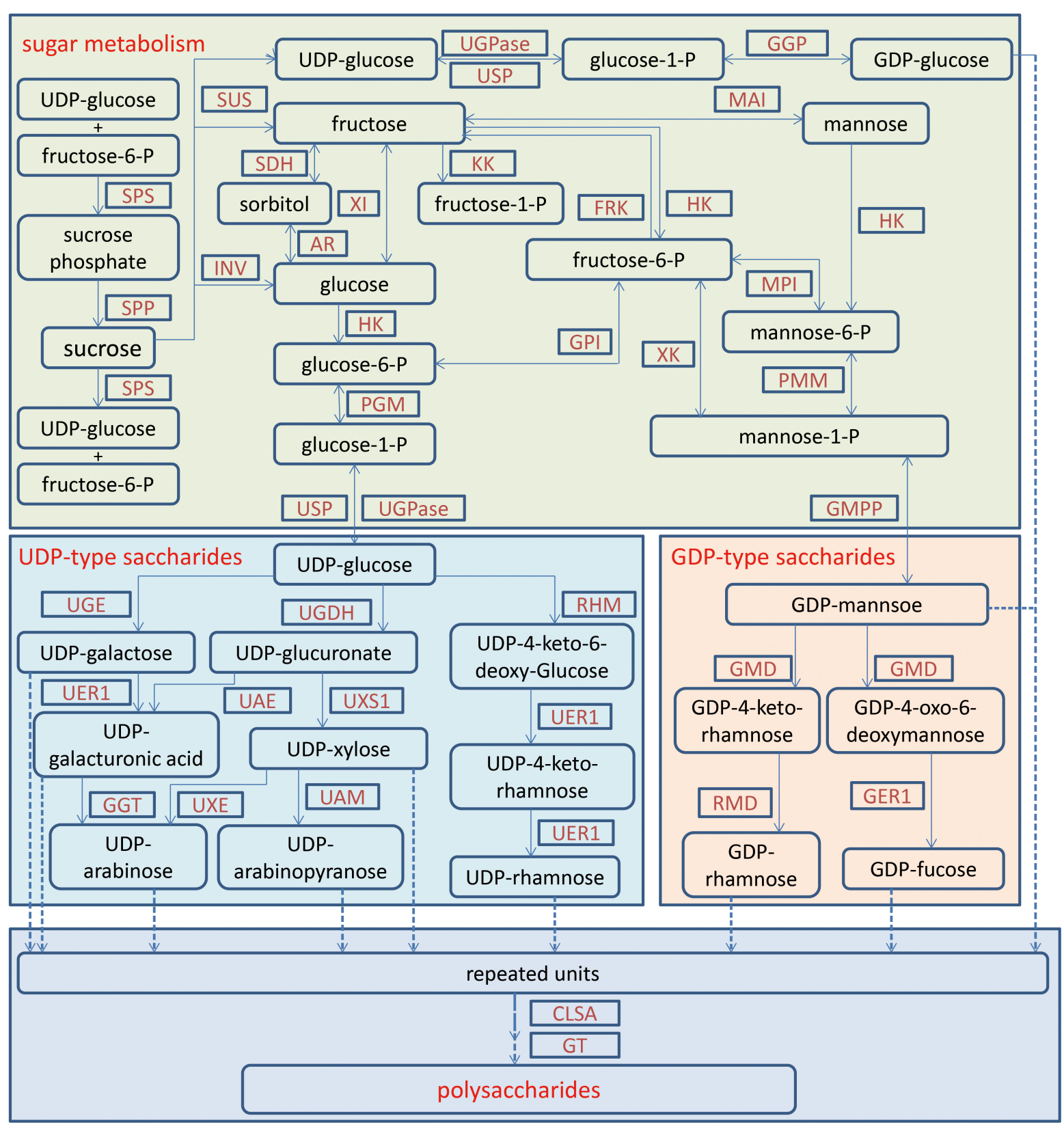

FIGURE 2 | The biosynthetic pathway for polysaccharides in Dendrobium officinale. The biosynthesis of polysaccharides contains sugar metabolism, UDP-saccharide metabolism, GDP-saccharide metabolism and polysaccharide formation. AR, aldose reductase; CSLA, cellulose synthase-like A; FRK, fructokinase; GAE, UDP-glucose A-4-epimerase; GER1, GDP-4-keto-6-deoxy-D-mannose-3,5-epimerase-4-reductase; GGP, glucose-1-phosphate guanylyltransferase; GGT, UDP-galacturonate decarboxylase; GMD, GDP-D-mannose-4,6-dehydratase; GMPP, mannose-1-phosphate guanylyl transferase; GPI, glucose-6-phosphate isomerase; GT, glycosyltransferases; HK, hexokinase; INV, invertase; KK, ketohexokinase; MAl, mannose isomerase; MPI, mannose-6-phosphate isomerase; PGM, phosphoglucomutase; PMM, phosphomannomutase; RHM, UDP-glucose 4, 6-dehydratase; RMD, GDP-4-dehydro-D-rhamnose reductase; SDH, sorbitol dehydrogenase; SPP, sucrose phosphate phosphatase; SPS, sucrose phosphate synthase; SuS, sucrose synthase; TSTA3, GDP-I-fucose synthase; UAE, UDP-glucuronate 4-epimerase; UAM, UDP-arabinopyranose mutase; UER1, 3,5-epimerase/4-reductase; UGDH, UDP-glucose dehydrogenase; UGE, UDP-glucuronate 4-epimerase; UGPase, UDP-glucose pyrophosphorylase; USP, UDP-sugar pyrophosphorylase; UXE, UDP-arabinose 4-epimerase; UXS1, UDP-glucuronate decarboxylase; $\mathrm{XI}$, xylose isomerase; $\mathrm{XK}$, xylulokinase.

shikimate dehydrogenase, mevalonate kinase (MVK), and aminotransferases were mainly expressed in leaves, which could be the main reason for higher alkaloid content in leaves (Shen et al., 2017). Recently, 41 genes showing significant differences in expression levels were detected between PLBs and leaves, including genes in the strictosidine biosynthesis (Wang et al., 2021). The candidate genes encoding strictosidine $\beta$-D-Glucosidase, geissoschizine synthase and vinorine synthase 




in the alkaloid biosynthesis enzymes were first identified, which facilitates the analysis of its metabolite biosynthesis in future studies.

Polysaccharides in D. officinale are the most abundant active compounds in various tissues and adult stems show the highest content (He et al., 2015; Zhang J. et al., 2016; Shen et al., 2017). A metabolic pathway for fructose and mannose biosynthesis has been developed through comparative transcriptomic analysis of different tissues, including 44 genes (He et al., 2015; Figure 2). The expression patterns of eight CELLULOSE SYNTHASELIKE A (CSLA) genes were closely linked to the content of mannose in stems (He et al., 2015). Similarly, other putative genes responsible for higher polysaccharides in adult stems were identified, including mannose-1-phosphate guanylyltransferase, GDP-L-fucose synthase and mannose-6-phosphate isomerase (Shen et al., 2017). Using the PacBio sequencing technique, alternative splicing forms of two glycosyltransferase and four cellulose synthase genes have been detected, and two genes encoding SWEET and sucrose transporter showed higher expression in stems than in leaves (He et al., 2017). Although the biosynthesis for polysaccharides is complex, these studies provide hints for further investigation on the molecular mechanism of rich polysaccharides in $D$. officinale.

There are abundant phenols in D. officinale. Among them, flavonoids were found in leaves, stems, and roots of $D$. officinale, and the content in leaves was the highest (Yuan et al., 2020b). The biosynthetic pathway of flavonoids was proposed and contained 26 genes including chalcone synthase (CHS), flavanone 3-dioxygenase $(\mathrm{F} 3 \mathrm{H})$, dihydroflavonol reductase (DFR), flavonol synthase (FLS), trans-cinnamate 4-monooxygenase, leucoanthocyanidin dioxygenase (LAR), anthocyanidin reductase (ANR), and shikimate O-hydroxycinnamoyl transferase (Yuan et al., 2020b; Figure 3). DEGs were enriched in phenylpropanoid and flavonoid biosynthesis. Multiple structural genes in the flavonoid pathway were significantly upregulated in leaves, such as one LAR1, one DFR3, one $F 3 H$, and three CHS genes, which facilitate the accumulation of flavonoids in leaves and could be the main reason for different flavonoid contents among tissues. Recently, it has been reported that the contents of flavonoids and anthocyanins were different in the stems of $D$. officinale from three provinces (Lei et al., 2018; Ren et al., 2020). Different anthocyanin contents could result from the different activities of some key enzymes, such as hydroxyl cinnamomum acyltransferase 1, UDP-glycosyltransferase (UGT)-83A1, chalcone flavone isomerase-like, and UGT-88B1 serine carboxypeptidase-like 1 (Ren et al., 2020).

Exogenous application of plant hormones can induce accumulation of metabolites in medicinal plants, including in D. officinale. Treatments with methyl jasmonate (MeJA) and salicylic acid (SA) resulted in more active compounds 
TABLE 2 | A summary of transcriptomic reports in D. officinale.

\begin{tabular}{|c|c|c|c|}
\hline Tissues & $\begin{array}{l}\text { Treatments or } \\
\text { conditions }\end{array}$ & Research focus & References \\
\hline S & - & $\begin{array}{c}\text { Alkaloid, genetic } \\
\text { markers }\end{array}$ & $\begin{array}{l}\text { Guo et al., } \\
2013\end{array}$ \\
\hline$F, R, L$, and $S$ & - & Organ-specific study & $\begin{array}{l}\text { Meng et al., } \\
2016\end{array}$ \\
\hline$L, S, R$, and $F$ & - & $\begin{array}{c}\text { Polysaccharide } \\
\text { synthesis, alkaloid } \\
\text { synthesis }\end{array}$ & $\begin{array}{l}\text { Shen et al., } \\
2017\end{array}$ \\
\hline$S$ and $L$ & - & $\begin{array}{c}\text { Variant splicing, sugar } \\
\text { translocation }\end{array}$ & He et al., 2017 \\
\hline Eight tissues & - & Expression & $\begin{array}{l}\text { Zhang et al., } \\
\qquad 2017\end{array}$ \\
\hline$R, S$, and $L$ & - & Flavonoid biosynthesis & $\begin{array}{l}\text { Yuan et al., } \\
2020 b\end{array}$ \\
\hline$L, S, R$, and $F$ & - & Flowering & $\begin{array}{c}\text { Chen Y. et al., } \\
2017\end{array}$ \\
\hline$L, R, S$, and $F$ & - & $\begin{array}{c}\text { Polysaccharide } \\
\text { synthesis }\end{array}$ & $\begin{array}{l}\text { Zhang G. Q. } \\
\text { et al., } 2016\end{array}$ \\
\hline PLB, L & - & Alkaloid synthesis & $\begin{array}{c}\text { Wang et al., } \\
2021\end{array}$ \\
\hline Seedlings & Juvenile and adult & $\begin{array}{c}\text { Polysaccharide } \\
\text { synthesis }\end{array}$ & $\begin{array}{l}\text { Zhang J. et al., } \\
2016\end{array}$ \\
\hline S & Four stages & $\begin{array}{c}\text { Mannan } \\
\text { polysaccharides }\end{array}$ & He et al., 2015 \\
\hline $\mathrm{R}$ & Cadmium stress & Stress response & $\begin{array}{l}\text { Jiang et al., } \\
2020\end{array}$ \\
\hline $\mathrm{L}$ & MeJA & $\begin{array}{l}\text { Accumulation of } \\
\text { alkaloids }\end{array}$ & $\begin{array}{c}\text { Chen et al., } \\
2019\end{array}$ \\
\hline Seedlings & Far-red light & Shade-avoidance & $\begin{array}{l}\text { Li H. et al., } \\
2021\end{array}$ \\
\hline Seeds & $\begin{array}{l}\text { Symbiotic and } \\
\text { asymbiotic }\end{array}$ & Germination & $\begin{array}{c}\text { Chen J. et al., } \\
2020\end{array}$ \\
\hline $\mathrm{L}$ & Drought & Drought stress & $\begin{array}{c}\text { Wan et al., } \\
2018\end{array}$ \\
\hline $\mathrm{L}$ & $\mathrm{CO}_{2}$ & $\begin{array}{l}\text { Crassulacean acid } \\
\text { metabolism }\end{array}$ & Zou et al., 2018 \\
\hline $\begin{array}{l}\text { Germinated } \\
\text { seeds }\end{array}$ & $\begin{array}{c}\text { Symbiotic and } \\
\text { asymbiotic seeds }\end{array}$ & Symbiotic association & $\begin{array}{c}\text { Wang et al., } \\
2018\end{array}$ \\
\hline$L$ & $\begin{array}{l}\text { Drought stress and } \\
\text { stress removal }\end{array}$ & Drought stress & Zou et al., 2018 \\
\hline Plants & SA & $\begin{array}{c}\text { Polysaccharide, } \\
\text { flavonoid, and alkaloid } \\
\text { synthesis }\end{array}$ & Niu et al., 2021 \\
\hline $\mathrm{R}$ & MeJA & Bibenzyl biosynthesis & $\begin{array}{l}\text { Adejobi et al., } \\
2021\end{array}$ \\
\hline S & Three provinces & Flavonoids & Lei et al., 2018 \\
\hline S & Three provinces & Anthocyanins & Ren et al., 2020 \\
\hline$S, L$, and $R$ & Three species & $\begin{array}{l}\text { Polysaccharide and } \\
\text { alkaloid synthesis }\end{array}$ & $\begin{array}{l}\text { Yuan et al., } \\
2020 a\end{array}$ \\
\hline Styles & $\begin{array}{l}\text { Non-, self-, and } \\
\text { cross-pollinated }\end{array}$ & Self-incompatibility & $\begin{array}{c}\text { Chen Y. et al., } \\
2021\end{array}$ \\
\hline
\end{tabular}

S, stems; L, leaves; F, flowers; $R$, roots; PLB, protocorm-like bodies; Eight tissues $L, S$, column, flower buds, lip, sepal, white root, and green root tip.

accumulated in various tissues (Jiao et al., 2018; Chen et al., 2019; Adejobi et al., 2021; Niu et al., 2021). After MeJA treatment the alkaloids were enhanced in leaves of $D$. officinale through upregulating expression levels of the multiple genes in the pathways of MVA and MEP, such as P450 genes, transaminase genes and methyltransferase (Chen et al., 2019). The bibenzyl compounds were mainly accumulated in the roots and greatly increased after MeJA treatment because some key genes involved in the flavonoid and bibenzyl pathways were abundantly expressed after treatment, including P450 and putative bibenzyl synthase genes (Adejobi et al., 2021; Figure 3). Presence of SA in the culture medium can increase the accumulation of active components in D. officinale seedlings including alkaloids, polysaccharides, and flavonoids (Niu et al., 2021). As many as 107 genes involved in biosynthesis of active components were upregulated. Among them, two key enzyme genes $\left(F 3^{\prime} H\right.$ and $\left.D F R\right)$ involved in the anthocyanin synthesis were significantly upregulated, which may result in color differences between individual plants (Niu et al., 2021). These studies could help us improve the contents of bioactive compounds and supply information for further functional investigation of putative genes.

Transcriptomics can also be used to analyze other physiological processes. In nature D. officinale generally forms few seeds potentially because of self-incompatibility. A total of 41 putative genes involved in self-incompatibility were identified, including six $\mathrm{Ca}^{2+}$ signal genes and $11 \mathrm{~S}$-locus receptor kinase (SRK) related genes (Chen Y. et al., 2021), which supply helpful insights for genetic mechanisms and preservation of D. officinale resources. Moreover, D. officinale seeds are small and have limited energy reserves, and the symbiotic relationship between $D$. officinale and mycorrhizal fungi is beneficial for $D$. officinale. RNA-Seq showed that endogenous hormones play a vital role in the seed germination of $D$. officinale (Wang et al., 2018). The relatively low concentrations of JA, abscisic acid (ABA) and strigolactones (SLs) may promote the growth of D. officinale (Wang et al., 2018; Chen J. et al., 2020). After inoculation of mycorrhizal fungi, genes associated with gibberellic acid (GA) biosynthesis were upregulated, and GA3 may be a key signaling molecule for germination (Chen J. et al., 2020). These results provide valuable insights for orchid-fungal symbiosis and seed germination in Orchidaceae. Under cadmium stress, D. officinale showed regulatory responses in roots through metal transporters, sulfate glutathione metabolism, cell wall metabolism, and phenylpropanoid metabolism (Jiang et al., 2020). Furthermore, as an epiphytic plant, D. officinale shows strong resistances to environments and can partly use crassulacean acid metabolism under stress conditions (Zhang et al., 2018; Zou et al., 2018). Although transcriptomic data on drought treatments have been obtained, further analysis still needs be performed to explore the mechanisms behind drought resistance (Wan et al., 2018; Zou et al., 2018).

As summarized above, many transcriptomic researches have been carried out in D. officinale, including various tissues, treatments or conditions. Tissue-specific patterns and key genes in the biosynthesis of bioactive compounds and responses to stresses have been extensively investigated. This research provides valuable molecular information for understanding these distinct physiological processes. At present, it is urgent to build a public, open database of transcriptional resources for $D$. officinale as the established database for Arabidopsis thaliana (The Arabidopsis 
Information Resource, TAIR), which can provide easy access to researchers, especially for those who understand the function of genes but are not familiar with bioinformatics tools. In the future, the newest sequencing techniques, especially the third generation platform, should be used for further analysis, such as alternative splicing.

\section{PROTEOMIC RESEARCH IN DENDROBIUM OFFICINALE}

Because the changes of gene expression profiles at the mRNA level are not always the same as the changes at the protein level, proteomic research in plants can help to reveal the molecular mechanisms of plant growth, development, metabolites and responses to environments. At present, the methods in proteomics include isobaric tags for relative and absolute quantification (iTRAQ), data-independent acquisition (DIA), and tandem mass tags (TMT), which can detect protein expression levels, post-translational modifications and proteinprotein interactions of all proteins.

Proteomic research into $D$. officinale is a new avenue of research. The first report on $D$. officinale focused on analysis of root induced by Mycena dendrobii (Xu et al., 2015), which shows important proteins related to defense and stress responses, the formation of mycorrhizal fungi and the biosynthesis of bioactive components. These data explain why $M$. dendrobii can promote the growth of $D$. officinale seedlings. The lysine succinylation sites on $D$. officinale proteins were identified (Feng et al., 2017), and five key enzymes in the glycolytic pathway exhibit succinylation of lysine, which will promote understanding the functions of lysine succinylation in plants. Recently, to discriminate D. officinale from other Dendrobium species, among 343 measurable peptides, 29 peptides were chosen as putative biomarkers, and the short peptide ALGLELDLSER can be a biomarker for the identification of $D$. officinale plants from different geographical areas (Fang et al., 2020).

The aim of proteomics research in medicinal plants is usually to evaluate the active components and proteomes across different samples and dissect the molecular mechanisms of bioactive compounds. Compared with the gene expression profiles at the mRNA level, protein expression patterns can more reliably and accurately reflect the expression level of key enzymes in the biosynthesis pathways of active components. The deep research needs to be performed on the proteome of $D$. officinale, especially on the changes that occur at different stages and under various stresses.

\section{METABOLOMIC RESEARCH IN DENDROBIUM OFFICINALE}

As metabolites represent the final products of gene expression and protein function, they can reflect changes in plant growth, development and responses to environments. Metabolomics has become a widely used tool in plant research, including wide-targeted metabolomics and targeted metabolomics.
Due to bioactive functions of multiple metabolites in D. officinale, it is of great significance to analyze those metabolites (Table 3).

The different metabolites in different Dendrobium species can be used to discriminate between them. There were 11 secondary metabolites that were considered as biomarkers of $D$. officinale and Dendrobium huoshanense (Jin et al., 2016). Moreover, in these two species, as many as 133 nitrogenous compounds were identified, and allyl alkaloid is an important medicinal component worth further investigation (Song et al., 2020). For D. officinale plants, the third year is the most suitable harvest time based on the accumulation of metabolites (Jin et al., 2016). The metabolites vary often in different tissues of $D$. officinale. The stems were composed of higher levels of multiple metabolites than the leaves, while leaves included higher concentrations levels of polyphenols and lipids (Cao et al., 2019). Moreover, 649 different metabolites were found in the stem and leaf of 6-monthold D. officinale (Cao et al., 2019), including organic acids, amino acids, nucleotides, and flavonoids.

Multiple factors influence the accumulation of metabolites. There were 101 volatile compounds detected in stems of D. officinale from four regions, and some distinctive compounds were found in certain region ( $\mathrm{Hu}$ et al., 2020). UV-B radiation can induce accumulation of polysaccharides, alkaloids, and flavonoids in D. officinale stem (Chen Y. et al., 2020). The stems of $D$. officinale grown on different substrates contained significantly different metabolites (Zuo et al., 2020), and the mainly changed metabolites were the flavonoids. The stems from plants grown in pine bark showed a higher content of flavonoids, which provides the practical basis of substrate selection for D. officinale plants. Furthermore, the accumulation of alkaloids was increased when protocorm-like bodies of D. officinale were treated with tryptophan (secologanin) and MeJA (Jiao et al., 2018), and among them changes in 29 metabolites were confirmed, including carapanaubine, a kind of TIAs.

TABLE 3 | A summary of metabolomic studies in D. officinale.

\begin{tabular}{|c|c|c|c|}
\hline Materials & $\begin{array}{c}\text { Treatments or } \\
\text { conditions }\end{array}$ & Method & References \\
\hline Stems & $\begin{array}{c}\text { Three different } \\
\text { substrates }\end{array}$ & UPLC-MS/MS & Zuo et al., 2020 \\
\hline $\begin{array}{l}\text { Protocorm-like } \\
\text { bodies }\end{array}$ & $\begin{array}{c}\text { Precursors and } \\
\text { methyl } \\
\text { jasmonate }\end{array}$ & $\begin{array}{l}\text { GC-MS, } \\
\text { LC-MS }\end{array}$ & Jiao et al., 2018 \\
\hline Stems & $\begin{array}{l}\text { Different growth } \\
\text { years of two } \\
\text { species }\end{array}$ & GC-MS & Jin et al., 2016 \\
\hline Stems & UV-B treatment & UPLC-MS/MS & $\begin{array}{c}\text { Chen Y. et al., } \\
2020\end{array}$ \\
\hline Caulis & $\begin{array}{c}\text { Two } \\
\text { Dendrobium } \\
\text { plants }\end{array}$ & LC-MS & $\begin{array}{l}\text { Song et al., } \\
2020\end{array}$ \\
\hline $\begin{array}{l}\text { Leaves and } \\
\text { stems }\end{array}$ & - & LC-ESI-MS/MS & $\begin{array}{l}\text { Cao et al., } \\
2019\end{array}$ \\
\hline Stems & $\begin{array}{l}\text { Different } \\
\text { regions }\end{array}$ & GC-MS & Hu et al., 2020 \\
\hline
\end{tabular}


TABLE 4 | A summary of omics integration in D. officinale.

\begin{tabular}{|c|c|c|c|c|}
\hline Samples & $\begin{array}{l}\text { Treatments or } \\
\text { conditions }\end{array}$ & $\begin{array}{c}\text { Combinations of } \\
\text { omics }\end{array}$ & $\begin{array}{l}\text { Research } \\
\text { focus }\end{array}$ & References \\
\hline Stems & $\begin{array}{c}\text { Purple and } \\
\text { normal varieties }\end{array}$ & $\begin{array}{c}\text { UPLC-ESI-MS/MS, } \\
\text { RNA-seq }\end{array}$ & Pigmentation & $\begin{array}{c}\text { Zhan et al., } \\
2020\end{array}$ \\
\hline Flowers & $\begin{array}{c}\text { Buds and } \\
\text { opened flower }\end{array}$ & $\begin{array}{l}\text { RNA-seq, } \\
\text { HPLC-MS }\end{array}$ & $\begin{array}{c}\text { Flower } \\
\text { development }\end{array}$ & He et al., 2020 \\
\hline Seeds & $\begin{array}{l}\text { Asymbiotic and } \\
\text { symbiotic at } \\
\text { different stages }\end{array}$ & RNA-Seq, iTRAQ & $\begin{array}{l}\text { Symbiotic } \\
\text { germination }\end{array}$ & $\begin{array}{c}\text { Chen J. et al., } \\
2017\end{array}$ \\
\hline Leaves & $\begin{array}{c}\text { at } 0^{\circ} \mathrm{C} \text { and at } \\
20^{\circ} \mathrm{C}\end{array}$ & GC-MS, RNA-seq & $\begin{array}{c}\text { Cold } \\
\text { acclimation }\end{array}$ & Wu et al., 2016 \\
\hline Leaves & $\mathrm{NaCl}$ treatment & $\begin{array}{l}\text { RNA-seq, } \\
\text { LC-MS/MS }\end{array}$ & Salt stress & $\begin{array}{l}\text { Zhang et al., } \\
\qquad 2021\end{array}$ \\
\hline Stems & Fungus MF23 & $\begin{array}{l}\text { RNA-seq, } \\
\text { UHPLC-LTQ }\end{array}$ & $\begin{array}{l}\text { Fungus on } \\
\text { growth }\end{array}$ & $\begin{array}{l}\text { Shan et al., } \\
2021\end{array}$ \\
\hline Flowers & Two cultivars & RNA-seq, GC-MS & $\begin{array}{c}\text { Volatile } \\
\text { terpenoids }\end{array}$ & $\begin{array}{l}\text { Li N. et al., } \\
2021\end{array}$ \\
\hline
\end{tabular}

To date, metabolomics studies into $D$. officinale remain limited. However, with technological development, more attention could be placed on this area because it is home to important medicinal properties in need of elucidation. Metabolomics studies not only provide us insights of metabolite accumulation under different conditions, but also important potential management methods that can improve the contents of target metabolites. Moreover, although wide-targeted metabolomics can provide huge information regarding metabolites, more focuses needs be paid to the active compounds through targeted metabolomics in D. officinale, which can provide more accurate information for certain type of metabolites.

\section{INTEGRATION RESEARCH OF MULTI-OMICS IN DENDROBIUM OFFICINALE}

The process of plant growth and development is complex and changeable. The plant status is the result of interactions between the genetic information and environmental factors. Integration of multi-omics can provide more comprehensive information to understand the dynamic changes and the potential mechanisms of various physiological processes. At present, there are some reports on the integration of different omics in D. officinale as shown in Table 4. Mainly, transcriptomics and metabolomics are associated to dissect changes in gene expression and metabolite accumulation in different tissues or under special conditions, which can be used to construct a regulatory network.

The combination of metabolomic and transcriptomic analysis has also widely been used to analyze various physiological processes in D. officinale, such as flower development (He et al., 2020), cold acclimation (Wu et al., 2016), stem color (Zhan et al., 2020), salt stress (Zhang et al., 2021), and flower volatile terpenoids ( $\mathrm{Li} \mathrm{N}$. et al., 2021). Some key factors have been identified to explain why the metabolites were changed, including the key enzymes in the biosynthetic pathway and potential transcription factors (Li N. et al., 2021). For example, the increase in content of delphinidin and quercetin derivatives was responsible for the purple stem of $D$. officinale and $\mathrm{F} 3 \mathrm{H}$ and leucoanthocyanidin dioxygenase $(L D O X)$ genes were found to be highly expressed (Zhan et al., 2020). Through analysis of seed germination by transcriptomics and proteomics (Chen J. et al., 2017), 32 genes showed consistent levels at mRNA and protein level and some of them were related to lipid and glycometabolism, which may be induced by fungi during germination, offering valuable insights into seed germination for orchid plants. Mycena sp. can promote the growth of $D$. officinale due to better nitrogen uptake and $\mathrm{NH}_{4}{ }^{+}$assimilation (Shan et al., 2021), which supplies the molecular basis for cultivation.

Reports on combination of different omics are presently limited in D. officinale. However, the ample advantages of the approach are amply demonstrated (Table 4). The molecular status of plants can comprehensively be reflected at different levels, which is conducive to screening for trait-related genes and for further investigation.

\section{PERSPECTIVES}

Due to its high medicinal and economic values, D. officinale has attracted significant attention from researchers. Thanks to the rapid development of sequencing and analysis methods, close relationships have been built between traditional Chinese medicine and genetic basis (Tables 1-3). Various biological processes of medicinal plants including $D$. officinale are now increasingly being investigated. Among them, the omics methods have been considered as a powerful tool to obtain a huge amount of information, including gene sequences, gene expression patterns, protein expression profiles, and metabolites in $D$. officinale. Therefore, in-depth studies of the molecular mechanisms underlying the biological processes are now possible and credible, such as the accumulation of active components. In recent years, many breakthroughs have been made in the study of $D$. officinale. Of particular note, the third version of the $D$. officinale genome at the chromosome level has recently been reported (Niu et al., 2021), which facilitates future genomic research. High-quality genomic data of $D$. officinale can have a vital influence on further studies into the accumulation and regulation of active components and molecular breeding of D. officinale. Transcriptomics of $D$. officinale is mainly focused on the key enzyme genes in the pathway of active component synthesis and analyzing its metabolic pathway.

Although great strides have been made in deepening our understanding of $D$. officinale, limitations remain. First, many genes related to active components in $D$. officinale have been identified by genomic and transcriptomic studies (Figures 13 ), but the functions of most these genes have not been confirmed through functional investigation, and their regulatory mechanisms have not yet been elucidated. For example, MeJA has been shown to induce the accumulation of polysaccharides, 


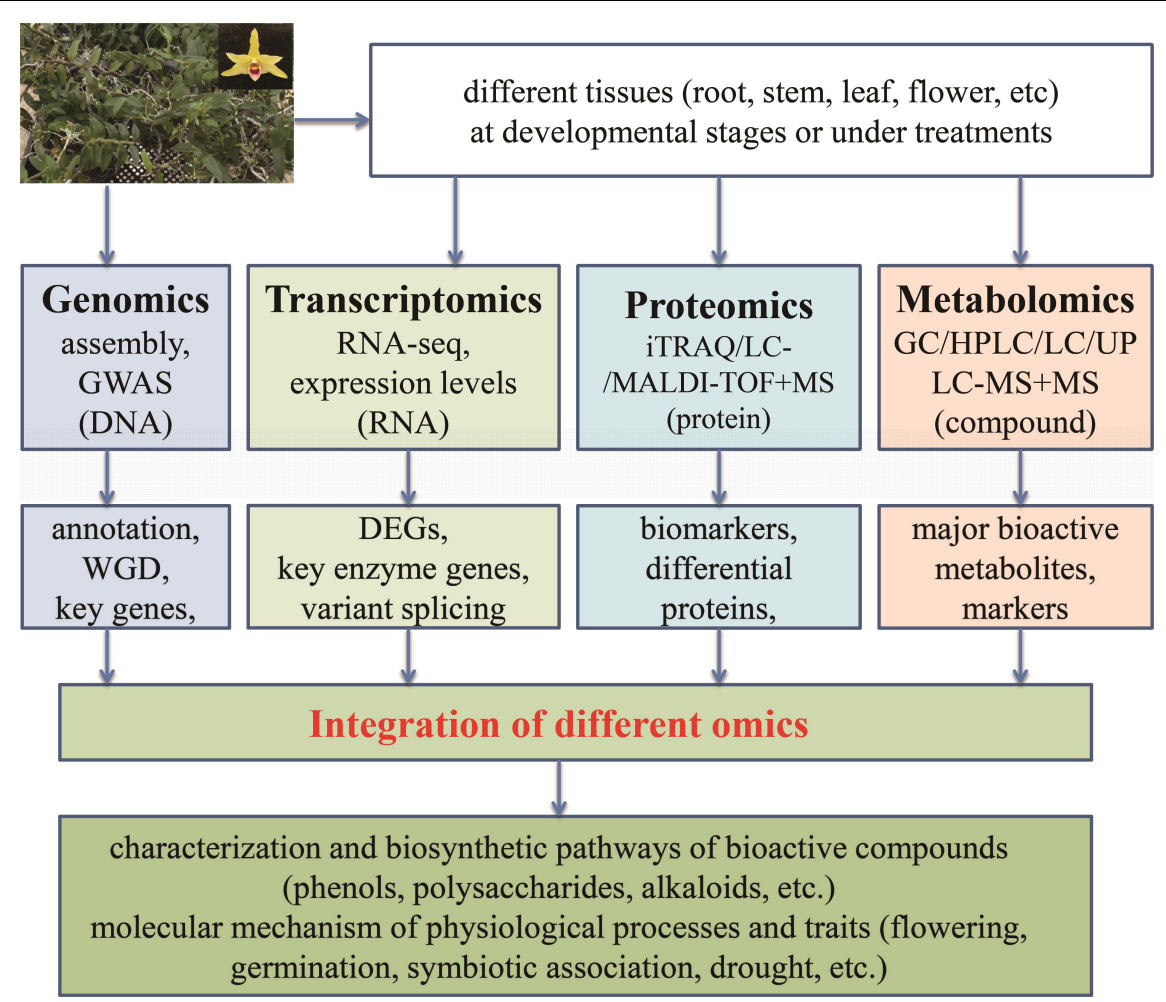

FIGURE 4 | Application of multiple omics in Dendrobium officinale. The integration of data from different levels (DNA, RNA, protein, and metabolites) can make it feasible and reliable to discover the potentially key factors (transcription factor, gene, protein, or compound) responsible for bioactive compounds as well as physiological processes and traits.

flavonoids, and alkaloids in D. officinale (Yuan et al., 2017; Jiao et al., 2018; Chen et al., 2019; Adejobi et al., 2021). However, the key factors in the JA signal pathway still need to be identified and clarified. Second, $D$. officinale plants generally live in extremely harsh environments and show strong drought resistance, the molecular mechanism of which is still limitedly clarified. There is a large amount of transcriptomic data available on D. officinale treated under different drought conditions (Wan et al., 2018; Zou et al., 2018). However, these data have not yet been fully and deeply analyzed. Therefore, there is an urgent need to excavate the data to reveal the molecular mechanisms of drought resistance in D. officinale.

In order to promote further researches into D. officinale, attentions should be paid to the following work in the future: (1) Although current omics technologies have been used to study $D$. officinale, there are limitedly comprehensive multiomics studies. Obviously, one single omics method such as transcriptomics, proteomics, or metabolomics cannot satisfy the necessary deep research into $D$. officinale. Therefore, it is imperative to study the mechanisms that underlie biosynthesis and the regulation of active compounds in $D$. officinale via the integration of multi-omics (Figure 4). In particular, the combination of proteomics and metabolomics has not yet been carried out for D. officinale. (2) The genuine resource of medicinal plants is essential for their functions, including D. officinale. Therefore, it is important to comprehensively investigate why differences exist in active compounds between plants from different cultivation regions or conditions at the genome, mRNA and protein levels, which can provide propitiate conditions for metabolite accumulation. (3) Based on the whole genome of $D$. officinale, resequencing of different germplasm resources can help to screen the key genes responsible for interesting traits for further functional dissection. In particular, studies on the mechanisms of biosynthesis and regulation of growth and development of active ingredients should be continued. (4) Although some genes belonging to the biosynthetic pathway of the main active ingredients in D. officinale have been identified, the accurate functions of only a few genes have been confirmed. Studies on key genes related to the biosynthesis of active components, growth regulation, and stress resistance of $D$. officinale require further investigated. Recently, the potential transformation system for Dendrobium plants was reported (Li Y. et al., 2021), which could benefit the functional investigation into $D$. officinale.

\section{AUTHOR CONTRIBUTIONS}

AL: conceptualization and writing - review and revising. YueW, OA, YT, and YuhW: literature search and data analysis. YueW: writing - original draft. All authors have read and agreed to the published version of the manuscript. 


\section{FUNDING}

We are grateful for funds supported from the National Natural Science Foundation of China (grant number 31701465), Yunnan Provincial Science and Technology Department (grant number 202101AT070189), and Beijing DR PLANT Biotechnology Co., Ltd. (E0514832C1). This study received funding from Beijing DR PLANT Biotechnology Co. Ltd. The funder was not involved in the study design, collection, analysis,

\section{REFERENCES}

Adejobi, O. I, Guan, J., Yang, L., Hu, J. M., Yu, A., Muraguri, S., et al. (2021). Transcriptomic analyses shed light on critical genes associated with bibenzyl biosynthesis in Dendrobium officinale. Plants 10:633. doi: 10.3390/ plants 10040633

Cao, H., Ji, Y., Li, S., Lu, L., Tian, M., Yang, W., et al. (2019). Extensive metabolic profiles of leaves and stems from the medicinal plant dendrobium officinale kimura et migo. Metabolites 9:215. doi: 10.3390/metabo9100215

Chen, J., Liu, S. S., Kohler, A., Yan, B., Luo, H. M., Chen, X. M., et al. (2017). iTRAQ and RNA-Seq analyses provide new insights into regulation mechanism of symbiotic germination of Dendrobium officinale Seeds (Orchidaceae). J. Proteome Res. 16, 2174-2187. doi: 10.1021/acs.jproteome.6b 00999

Chen, J., Yan, B., Tang, Y., Xing, Y., Li, Y., Zhou, D., et al. (2020). Symbiotic and asymbiotic germination of Dendrobium officinale (Orchidaceae) respond differently to exogenous gibberellins. Int. J. Mol. Sci. 21:6104. doi: 10.3390/ ijms21176104

Chen, W., Lu, J., Zhang, J. Wu, J., Yu, L., Qin, L., et al. (2021). Traditional Uses, phytochemistry, pharmacology, and quality control of dendrobium officinale kimura et. migo. Front. Pharmacol. 12:726528. doi: 10.3389/fphar.2021.726528

Chen, Y., Hu, B., Zhang, F., Luo, X., and Xie, J. (2021). Cytological observation and transcriptome comparative analysis of self-pollination and cross-pollination in dendrobium officinale. Genes 12:432. doi: 10.3390/genes 12030432

Chen, Y., Shen, Q., Lin, R., Zhao, Z., Shen, C., and Sun, C. (2017). De novo transcriptome analysis in Dendrobium and identification of critical genes associated with flowering. Plant Physiol. Biochem. 119, 319-327. doi: 10.1016/j. plaphy.2017.09.008

Chen, Y., Shen, Q., Lv, P., and Sun, C. (2020). Comparative metabolomic analyses of Dendrobium officinale Kimura et Migo responding to UV-B radiation reveal variations in the metabolisms associated with its bioactive ingredients. PeerJ 8:e9107. doi: 10.7717/peerj.9107

Chen, Y., Wang, Y., Lyu, P., Chen, L., Shen, C., and Sun, C. (2019). Comparative transcriptomic analysis reveal the regulation mechanism underlying MeJAinduced accumulation of alkaloids in Dendrobium officinale. J. Plant Res. 132, 419-429. doi: 10.1007/s10265-019-01099-6

Fang, C., Xin, G. Z., Wang, S. L., Wei, M. M., Wu, P., Dong, X. M., et al. (2020). Discovery and validation of peptide biomarkers for discrimination of Dendrobium species by label-free proteomics and chemometrics. J. Pharm. Biomed. Anal. 182:113118. doi: 10.1016/j.jpba.2020.113118

Feng, S., Jiao, K., Guo, H., Jiang, M., Hao, J., Wang, H., et al. (2017). Succinylproteome profiling of Dendrobium officinale, an important traditional Chinese orchid herb, revealed involvement of succinylation in the glycolysis pathway. BMC Genomics 18:598. doi: 10.1186/s12864-017-3978-x

Guo, X., Li, Y., Li, C., Luo, H., Wang, L., Qian, J., et al. (2013). Analysis of the Dendrobium officinale transcriptome reveals putative alkaloid biosynthetic genes and genetic markers. Gene 527, 131-138. doi: 10.1016/j.gene.2013.05.073

He, C., Liu, X., Teixeira da Silva, J. A., Liu, N., Zhang, M., and Duan, J. (2020). Transcriptome sequencing and metabolite profiling analyses provide comprehensive insight into molecular mechanisms of flower development in Dendrobium officinale (Orchidaceae). Plant Mol. Biol. 104, 529-548. doi: 10. 1007/s11103-020-01058-Z

He, C., Zhang, J., Liu, X., Zeng, S., Wu, K., Yu, Z., et al. (2015). Identification of genes involved in biosynthesis of mannan polysaccharides in Dendrobium interpretation of data, the writing of this article or the decision to submit it for publication. All authors declare no other competing interests.

\section{ACKNOWLEDGMENTS}

We appreciate Austin G. Smith (ICRAF) for language improvement.

officinale by RNA-seq analysis. Plant Mol. Biol. 88, 219-231. doi: 10.1007/ s11103-015-0316-z

He, L., Fu, S., Xu, Z., Yan, J., Xu, J., Zhou, H., et al. (2017). Hybrid Sequencing of Full-Length cDNA Transcripts of Stems and Leaves in Dendrobium officinale. Genes 8:257. doi: 10.3390/genes8100257

Hu, J., Huang, W., Zhang, F., Luo, X., Chen, Y., and Xie, J. (2020). Variability of volatile compounds in the medicinal plant dendrobium officinale from different regions. Molecules 25:5046. doi: 10.3390/molecules25215046

Jiang, W., Wu, Z., Wang, T., Mantri, N., Huang, H., Li, H., et al. (2020). Physiological and transcriptomic analyses of cadmium stress response in Dendrobium officinale seedling. Plant Physiol. Biochem. 148, 152-165. doi: 10. 1016/j.plaphy.2020.01.010

Jiao, C., Song, C., Zheng, S., Zhu, Y., Jin, Q., Cai, Y., et al. (2018). Metabolic profiling of dendrobium officinale in response to precursors and methyl jasmonate. Int. J. Mol. Sci. 19:728. doi: 10.3390/ijms19030728

Jin, Q., Jiao, C., Sun, S., Song, C., Cai, Y., Lin, Y., et al. (2016). Metabolic analysis of medicinal dendrobium officinale and dendrobium huoshanense during different growth years. PLoS One. 11:e0146607. doi: 10.1371/journal.pone.0146607

Lei, Z., Zhou, C., Ji, X., Wei, G., Huang, Y., Yu, W., et al. (2018). Transcriptome Analysis Reveals genes involved in flavonoid biosynthesis and accumulation in Dendrobium catenatum From Different Locations. Sci. Rep. 8:6373. doi: 10.1038/s41598-018-24751-y

Li, H., Ye, W., Wang, Y., Chen, X., Fang, Y., and Sun, G. (2021). RNA sequencingbased exploration of the effects of far-red light on lncRNAs involved in the shade-avoidance response of D. officinale. PeerJ 9:e10769. doi: 10.7717/peerj. 10769

Li, N., Dong, Y., Lv, M., Qian, L., Sun, X., Liu, L., et al. (2021). Combined analysis of volatile terpenoid metabolism and transcriptome reveals transcription factors related to terpene synthase in two cultivars of dendrobium officinale flowers. Front. Genet. 12:661296. doi: 10.3389/fgene.2021.661296

Li, Y., Zhang, B., and Yu, H. (2021). Kilobase-scale genomic deletion of DOTFL1 in dendrobium orchids. J. Genet. Genomics 2021:8. doi: 10.1016/j.jgg.2021.07.008

Meng, Y., Yu, D., Xue, J., Lu, J., Feng, S., Shen, C., et al. (2016). A transcriptomewide, organ-specific regulatory map of Dendrobium officinale, an important traditional Chinese orchid herb. Sci. Rep. 6:18864. doi: 10.1038/srep 18864

Ng, T. B., Liu, J., Wong, J. H., Ye, X., WingSze, S. C., Tong, Y., et al. (2012). Review of research on Dendrobium, a prized folk medicine. Appl. Microbiol. Biotechnol. 93, 1795-1803. doi: 10.1007/s00253-011-3829-7

Niu, Z., Zhu, F., Fan, Y., Li, C., Zhang, B., Zhu, S., et al. (2021). The chromosome-level reference genome assembly for Dendrobium officinale and its utility of functional genomics research and molecular breeding study. Acta Pharmaceutica Sinica B. 2021:19. doi: 10.1016/j.apsb.2021.01.019

Ren, Z., Qiu, F., Wang, Y., Yu, W., Liu, C., Sun, Y., et al. (2020). Network analysis of transcriptome and lc-ms reveals a possible biosynthesis pathway of anthocyanins in dendrobium officinale. Biomed. Res. Int. 2020:6512895. doi: $10.1155 / 2020 / 6512895$

Shan, T., Zhou, L., Li, B., Chen, X., Guo, S., Wang, A., et al. (2021). The plant growth-promoting fungus MF23 (Mycena sp.) increases production of dendrobium officinale (orchidaceae) by affecting nitrogen uptake and $\mathrm{NH}_{4}{ }^{+}$ Assimilation. Front. Plant Sci. 12:693561. doi: 10.3389/fpls.2021.693561

Shen, C., Guo, H., Chen, H., Shi, Y., Meng, Y., Lu, J., et al. (2017). Identification and analysis of genes associated with the synthesis of bioactive constituents in Dendrobium officinale using RNA-Seq. Sci. Rep. 7:187. doi: 10.1038/s41598017-00292-8 
Song, C., Jiao, C., Jin, Q., Chen, C., Cai, Y., and Lin, Y. (2020). Metabolomics analysis of nitrogen-containing metabolites between two Dendrobium plants. Physiol. Mol. Biol. Plants 26, 1425-1435. doi: 10.1007/s12298-020-00822-1

Tang, H., Zhao, T., Sheng, Y., Zheng, T., Fu, L., and Zhang, Y. (2017). Dendrobium officinale kimura et migo: a review on its ethnopharmacology, phytochemistry, pharmacology, and industrialization. Evid. Based Comp. Alter. Med. 2017:7436259. doi: 10.1155/2017/7436259

Wan, X., Zou, L. H., Zheng, B. Q., Tian, Y. Q., and Wang, Y. (2018). Transcriptomic profiling for prolonged drought in Dendrobium catenatum. Sci. Data 5:180233. doi: $10.1038 /$ sdata.2018.233

Wang, F., and Shi, H. (2019). Research on the development of dendrobium candidum industry in china. China Foresty Econ. 3, 88-90.

Wang, T., Song, Z., Wang, X., Xu, L., Sun, Q., and Li, L. (2018). Functional insights into the roles of hormones in the dendrobium officinale-tulasnella sp. germinated seed symbiotic association. Int. J. Mol. Sci. 19:3484. doi: 10.3390/ ijms 19113484

Wang, Y. H. (2021). Traditional uses, chemical constituents, pharmacological activities, and toxicological effects of Dendrobium leaves: a review. J. Ethnopharmacol. 270:113851. doi: 10.1016/j.jep.2021.113851

Wang, Z., Jiang, W., Liu, Y., Meng, X., Su, X., Cao, M., et al. (2021). Putative genes in alkaloid biosynthesis identified in Dendrobium officinale by correlating the contents of major bioactive metabolites with genes expression between Protocorm-like bodies and leaves. BMC Genomics 22:579. doi: 10.1186/s12864021-07887-6

Wang, Z., Zhao, M., Cui, H., Li, J., and Wang, M. (2020). Transcriptomic landscape of medicinal dendrobium reveals genes associated with the biosynthesis of bioactive components. Front. Plant Sci. 11:391. doi: 10.3389/fpls.2020.00391

Wu, Z. G., Jiang, W., Chen, S. L., Mantri, N., Tao, Z. M., and Jiang, C. X. (2016). Insights from the cold transcriptome and metabolome of dendrobium officinale: global reprogramming of metabolic and gene regulation networks during cold acclimation. Front. Plant Sci. 7:1653. doi: 10.3389/fpls.2016.01653

Xu, X. B., Ma, X. Y., Lei, H. H., Song, H. M., Ying, Q. C., Xu, M. J., et al. (2015). Proteomic analysis reveals the mechanisms of Mycena dendrobii promoting transplantation survival and growth of tissue culture seedlings of Dendrobium officinale. J. Appl. Microbiol. 118, 1444-1455. doi: 10.1111/jam.12781

Yan, L., Wang, X., Liu, H., Tian, Y., Lian, J., Yang, R., et al. (2015). The genome of dendrobium officinale illuminates the biology of the important traditional chinese orchid herb. Mol. Plant. 8, 922-934. doi: 10.1016/j.molp.2014.12.011

Yuan, Y., Zhang, B., Tang, X., Zhang, J., and Lin, J. (2020a). Comparative transcriptome analysis of different dendrobium species reveals active ingredients-related genes and pathways. Int. J. Mol. Sci. 21:861. doi: 10.3390/ijms21030861

Yuan, Y., Zhang, J., Liu, X., Meng, M., Wang, J., and Lin, J. (2020b). Tissue-specific transcriptome for Dendrobium officinale reveals genes involved in flavonoid biosynthesis. Genomics 112, 1781-1794. doi: 10.1016/j.ygeno.2019.10.010

Yuan, Z. Q., Zhang, J. Y., and Liu, T. (2017). Enhancement of polysaccharides accumulation in Dendrobium officinale by exogenously applied methyl jasmonate. Biological. Plantarum. 61, 438-444. doi: 10.1007/s10535-0160702-7

Zhan, X., Qi, J., Zhou, B., and Mao, B. (2020). Metabolomic and transcriptomic analyses reveal the regulation of pigmentation in the purple variety of Dendrobium officinale. Sci. Rep. 10:17700. doi: 10.1038/s41598-020-74789-0

Zhang, G. N., Bi, Z. M., Wang, Z. T., Xu, L. S., and Xu, G. J. (2003). Advances in studies on chemical constituents from plants of Dendrobium Sw. Chinese Trad. Herb. Drugs 34, S5-S8.

Zhang, G. Q., Liu, K. W., Li, Z., Lohaus, R., Hsiao, Y. Y., Niu, S. C., et al. (2017). The Apostasia genome and the evolution of orchids. Nature 381:549. doi: 10.1038/nature23897

Zhang, G. Q., Xu, Q., Bian, C., Tsai, W. C., Yeh, C. M., Liu, K. W., et al. (2016). The Dendrobium catenatum Lindl. genome sequence provides insights into polysaccharide synthase, floral development and adaptive evolution. Sci. Rep. 6:19029. doi: 10.1038/srep19029

Zhang, J., He, C., Wu, K., Teixeira da Silva, J. A., Zeng, S., Zhang, X., et al. (2016). Transcriptome analysis of dendrobium officinale and its application to the identification of genes associated with polysaccharide synthesis. Front. Plant Sci. 7:5. doi: 10.3389/fpls.2016.00005

Zhang, M., Yu, Z., Zeng, D., Si, C., Zhao, C., Wang, H., et al. (2021). Transcriptome and metabolome reveal salt-stress responses of leaf tissues from dendrobium officinale. Biomolecules 5, 736-736. doi: 10.3390/BIOM11050736

Zhang, S., Yang, Y., Li, J., Qin, J., Zhang, W., Huang, W., et al. (2018). Physiological diversity of orchids. Plant Divers 40, 196-208. doi: 10.1016/j.pld.2018.06.003

Zou, L. H., Wan, X., Deng, H., Zheng, B. Q., Li, B. J., and Wang, Y. (2018). RNA-seq transcriptomic profiling of crassulacean acid metabolism pathway in Dendrobium catenatum. Sci. Data 5:180252. doi: 10.1038/sdata.2018.252

Zuo, S. M., Yu, H. D., Zhang, W., Zhong, Q., Chen, W., Chen, W., et al. (2020). Comparative metabolomic analysis of dendrobium officinale under different cultivation substrates. Metabolites 10:325. doi: 10.3390/metabo10080325

Conflict of Interest: The authors declare that the research was conducted in the absence of any commercial or financial relationships that could be construed as a potential conflict of interest.

Publisher's Note: All claims expressed in this article are solely those of the authors and do not necessarily represent those of their affiliated organizations, or those of the publisher, the editors and the reviewers. Any product that may be evaluated in this article, or claim that may be made by its manufacturer, is not guaranteed or endorsed by the publisher.

Copyright (c) 2022 Wang, Tong, Adejobi, Wang and Liu. This is an open-access article distributed under the terms of the Creative Commons Attribution License (CC BY). The use, distribution or reproduction in other forums is permitted, provided the original author(s) and the copyright owner(s) are credited and that the original publication in this journal is cited, in accordance with accepted academic practice. No use, distribution or reproduction is permitted which does not comply with these terms. 